Advance Publication

\title{
The Journal of Veterinary Medical Science
}

Accepted Date: 25 October 2021

J-STAGE Advance Published Date: 12 November 2021

(C)2021 The Japanese Society of Veterinary Science

Author manuscripts have been peer reviewed and accepted for publication but have not yet been edited. 
1 Physiology

2 Full paper

3

4 Effect of oral administration of colostrum on inflammation in the udders of dairy

5 cows suffering from mastitis

6

7 Naoki Isobe $^{1)^{*}}$, Tomoyasu Kurose ${ }^{2)}$, Naoki Suzuki ${ }^{1)}$, Tomoko Koshiishi ${ }^{3)}$, Kazutoshi

8 Ueno $^{4)}$, Keiichi Hisaeda ${ }^{5)}$

9

$10{ }^{1)}$ Graduate School of Integrated Sciences for Life, Hiroshima University, Higashi-

11 Hiroshima, 739-8528, Japan

$12{ }^{2)}$ Hiroshima Agricultural Mutual Aid Association, Hiroshima 732-0052, Japan

$13{ }^{3)}$ Ehime Agricultural Mutual Aid Association, Seiyo, Ehime 797-1211, Japan

14 4) e-animal Corporation, Gifu, 501-2122, Japan

$15{ }^{5)}$ Farm Animal Veterinary Nursing, Okayama University of Science, Imabari 794-8555, 16 Japan

17

*Corresponding author: Naoki Isobe, Graduate School of Biosphere Science, Hiroshima

19 University, Higashi-Hiroshima 739-8528, Japan; Phone and fax number: +81-424-7993;

E-mail: niso@hiroshima-u.ac.jp.

21

22 Running head: Effects of colostrum on bovine mastitis 
Abstract

The present study was undertaken to examine whether oral administration of colostrum to mastitic cows reduced inflammation in the udder. Fifty milliliters of a colostrum whey product was administered orally daily for 3 days to cows suffering from mastitis. Milk was collected on day 0 and 7 of colostrum administration. For Experiment 1, milk from 11 udder quarters with high somatic cell counts (SCC) in four cows was used. SCC in milk decreased significantly after colostrum administration, whereas colostrum administration increased sodium and IgA concentrations significantly compared with those before administration. In Experiment 2, cows with clinical mastitis were divided into two groups, with and without colostrum administration, whereas all cows with subclinical mastitis were administered colostrum. Antibiotics were infused into the mammary gland from the first day of colostrum administration for 2-4 days. There was no significant decrease in SCC after colostrum administration in any group. However, udder firmness in both groups was reduced after administration regardless of colostrum administration. IgA concentration in both clinical mastitis groups was significantly increased after colostrum administration compared to that before administration, although there was no significant difference between them. These results suggest the possibility that oral administration of colostrum attenuates inflammation of the mammary gland. Further studies are required to examine the effect of colostrum more precisely using cows with subclinical and chronic mastitis and longer duration of colostrum administration.

Keywords: colostrum; immunoglobulin A; mastitis; somatic cell count 
Mastitis is an inflammatory disease of the udder and causes huge economic losses in the dairy industry. To date, antibiotics have been the only successful treatment for mastitis in domestic ruminants. However, as it is possible that resistant bacteria have emerged over time, other methods to treat mastitis are urgently required. various important components (nutrients and immunoglobulin etc.) for newborn calves. However, colostrum has been used as a supplement for non-neonate animals. When colostrum whey was administered orally to 6-month-old goats every day for 3 weeks, plasma cells were recruited into the labial gland more frequently, which then secreted more immunoglobulin A (IgA) into the saliva [11]. Colostrum whey administration in prepartum goats increases IgA concentration in postpartum milk, which elevates IgA concentration in kid plasma, resulting in better daily weight gain in kids [8]. These results suggest that colostrum supplementation must reinforce immune function in the digestive tract and mammary glands of non-neonates. bovine colostrum after oral inoculation with porcine diarrhea virus showed a reduction of clinical symptoms and mortality, as compared to sows who were not provided colostrum [17]. In dogs infected with canine distemper virus, fecal IgA levels were higher in those fed a bovine-colostrum-supplemented diet than in those that had not received bovine colostrum[16]. These results suggest that colostrum plays a role in supporting the recovery of infectious diseases. However, there are no reports on the use of colostrum in animals suffering from mastitis. Upregulation of immunity in the mammary gland after 
colostrum administration may weaken the symptoms of inflammation, and result in recovery from mastitis.

Therefore, the present study was undertaken to examine whether colostrum supplementation in cows suffering from mastitis suppresses the inflammation in udders.

\section{Materials and methods}

\section{Experimental animals and sampling}

The study was divided into two experiments. For Experiment 1, we used four Holstein Friesian cows, from a private farm in Hiroshima Prefecture who were suffering from clinical mastitis in at least one quarter (four udders $=16$ quarters). Milk was collected from all four udders and somatic cell counts (SCCs) were measured using a Countess Automated Cell Counter (Life Technologies Japan Ltd., Tokyo, Japan). Only milk samples with an SCC of more than $3 \times 10^{5}$ cells $/ \mathrm{ml}$ were used for analysis.

For experiment 2, 80 multiparous Holstein Friesian cows (80 quarters; 2-38 weeks postpartum) from 20 private dairy farms in Ehime prefecture were classified into the following three groups: acute clinical with and without colostrum $(n=49)$ and subclinical mastitis with colostrum $(n=31)$. Acute clinical mastitis was diagnosed based on systemic and local mammary symptoms, such as body temperature, appetite, heart rate, induration or swelling of the udder, and milk clotting. The cows with clinical mastitis were further divided into groups with and without colostrum administration ( $\mathrm{n}=19$ and 30). Subclinical mastitis was diagnosed when a positive reaction was observed in the California mastitis test (CMT) using a PL tester (Nippon Zenyaku Kogyo Co., Ltd., Fukushima, Japan) without systemic and mammary local symptoms. All cows with 
subclinical mastitis were administered colostrum.

Fifty milliliters of colostrum whey product (eCow; e-Animal Co., Gifu, Japan) was administered orally daily for 3 days to all cows in Experiment 1, whereas only the cows in the clinical (Cli+) and subclinical (Subclinical) mastitis groups were administered colostrum in Experiment 2 following manufactural instructions. In the control group of Experiment 2, cows with clinical mastitis did not receive any colostrum $($ Cli-, $\mathrm{n}=30)$. eCow is an ultra-filtrated bovine whey product, which includes molecules whose molecular masses are less than $10 \mathrm{kDa}$. In Experiment 2, enrofloxacin (Bytril; Elanco, Tokyo, Japan) was injected intravenously for 2-4 days from the day of the first eCow administration. However, no other treatment was applied to cows in both experiment. This study was conducted in accordance with the guidelines of the Guidance for Animal Experiments protocol of Hiroshima University, Japan (C19-4).

Udder hardness was measured just after milking using a Digital Shore Tire Durometer C Hardness Tester Rubber Meter (Cbaoheng Mxbaoheng Co., Zhejiang, China) in experiment 2. In the front mastitic quarter, 2 places (top and bottom) in the forward of quarter and 4 places at intervals of about $5 \mathrm{~cm}$ from the top and bottom of the side of quarter were measured and the average was calculated. In the rear mastitic quarter, 3 places at intervals of about $5 \mathrm{~cm}$ from the top to bottom in each side and behind of quarter were measured and the average was calculated.

Milk samples $(10 \mathrm{ml})$ were collected at 0 and 7 days after first colostrum administration in plastic tubes and stored immediately at $4{ }^{\circ} \mathrm{C}$. SCCs were measured using a Countess Automated Cell Counter (Life Technologies) based on previously reported methods [12]. Next, the remaining milk was centrifuged at $1,900 \times g$ for 5 min at $4{ }^{\circ} \mathrm{C}$. Skim milk was stored at $-20^{\circ} \mathrm{C}$ for enzyme immunoassay. 


\section{Sample analysis}

Sodium $(\mathrm{Na})$ concentration in the milk was measured using a LAQUAtwin meter

123 (Horiba Co., Kyoto, Japan) following the manufacturer's protocol. Competitive enzyme

124 immunoassays were performed to measure lingual antimicrobial peptide (LAP) concentrations in skim milk based on previously described methods [6]. IgA concentration in milk was measured using an IgA commercial kit (Bethyl Laboratories, Inc., Montgomery, TX, USA) based on previously described methods [11]. Samples were diluted 10 and 1000 times for LAP and IgA determination, respectively. Optical density was measured using a microplate reader (Multiskan FC Microplate type 357; Thermo

130 Fisher Scientific, Tokyo, Japan).

\section{Statistical analysis}

Data were analyzed using the PROC MIXED procedure in SAS (Version 9.4, SAS Institute Inc., Cary, NC, USA), using the t-test (Experiment 1) or two-way repeated measures analysis of variance (ANOVA) followed by the Tukey-Kramer test for multiple comparisons (Experiment 2). The model included the group, time, and the interaction

137 between group and time. Results are reported as means and standard errors of means 138 (SEM). Differences were considered significant at $\mathrm{p}<0.05$.

\section{Results}

In Experiment 1, out of 16 quarters (four cows), 11 quarters with high SCC (> 
concentration was significantly increased in milk after colostrum administration

144 compared with that before administration (Fig. 1). Increased IgA concentration in mastitic 145 milk was also observed after colostrum administration compared to that before 146 administration. However, there was no significant difference in the LAP concentration in 147 mastitic milk before and after administration of colostrum.

148 In Experiment 2, our results show that firmness was affected by both group and 149 time, but not by their interaction (Fig. 2A). The firmness of the udder in both Cli+ and 150 Cli-groups was higher than that in Subclinical group before colostrum administration. 151 However, firmness was significantly decreased in the Cli+ and Cli-groups after colostrum administration. Both the Cli+ and Cli- groups tended to have higher SCC in milk than that in the Subclinical group before and after colostrum administration (Fig. 2B). However, there was no significant decrease in SCC after colostrum administration in all groups. Both $\mathrm{Cli}+$ and Cli- groups had significantly higher $\mathrm{Na}$ concentration than that in the Subclinical group before and after colostrum administration (Fig. 2C). However, Na concentrations in the milk from all groups did not significantly decrease after colostrum administration compared with those before administration. Before colostrum administration, LAP concentration in milk was significantly higher in the Cli- but not

$160 \mathrm{Cli}+$ than that in the Subclinical group; however, there was no significant difference between groups before and after administration (Fig. 3A). Concentrations of IgA in both

$162 \mathrm{Cli}+$ and Cli- groups were significantly increased after colostrum administration compared to those before administration (Fig. 3B).

164

Discussion

166 The present study was undertaken to examine whether oral colostrum 
administration was effective in treating bovine mastitis. In Experiment 1, SCC decreased after colostrum administration. This indicates that colostrum administration weakens the inflammatory status of the mammary gland. To confirm this effect, we prepared a control group without colostrum administration in Experiment 2. In experiment 1, since there is a possibility that both udders of clinical and subclinical mastitis were examined, these different types of mastitis were classified in experiment 2. There was no significant difference in SCC between before and after colostrum administration all groups. The reason for the different results between Experiments 1 and 2 is unclear. The major difference between the test animals in the two experiments was that, in Experiment 2, mastitic cows were treated with antibiotics in addition to colostrum. This suggests that antibiotics negate the effect of colostrum, resulting in the tendency to decrease SCC in both Cli groups. Alternatively, the reason for this difference between Experiments 1 and 2 may be the different selection of milk (udder). In experiment 1, mastitis was defined as SCC of $3 \times 10^{5}$ cells $/ \mathrm{ml}$ of milk and not based on diagnosing the symptoms of clinical mastitis, whereas, in Experiment 2, udders were classified as clinical or subclinical based significantly compared to that before administration in both Cli groups in Experiment 2. 
that the changes in udder firmness were more drastic than those in SCC after colostrum administration. SCC denotes the number of leukocytes in milk, whereas firmness depends on the number of leukocytes in the connective tissue[1]. Therefore, the present results suggest that leukocytes in connective tissue disappear faster than those in milk during recovery from mastitis. colostrum. They concluded that colostrum stimulates the recruitment of plasma cells into the labial gland, which then secretes more IgA into the saliva. Isobe et al. [8] found that IgA concentrations in postpartum milk were higher in goats who received prepartum oral colostrum for 2 weeks than in those who did not. This indicates that colostrum administration induces recruitment of plasma cells not only in the labial gland but also in the mammary gland. In the present study, in Experiment 2, significantly higher IgA was achieved after colostrum administration in both Cli (-/+) groups than that before administration, although there was no significant difference between the two groups. Therefore, the present results do not seem to indicate the effective role of colostrum in IgA production. This difference in our results on IgA concentration levels and those by previous study [8] may be due to the difference in the duration of colostrum administration - colostrum was administered for only 3 days in the present study and

214 inflammatory status of the mammary gland. Goats with healthy mammary glands were 
used in the previous study [8], whereas cows with mastitis were used in our study. Since subclinical mastitis is weak inflammation compared with clinical mastitis, colostrum may be more suitable for treatment of subclinical mastitis compared with clinical mastitis. Many antimicrobial components, such as S100A7 [20], S100A8 [13], cathelicidin [19], and lactoferrin [18] play a role in defending the mammary glands against infection [4]. LAP is also an antibacterial component in milk and is secreted by mammary epithelial cells [5-7]. Intramammary infusion of lipopolysaccharides (LPS) elevates LPS concentration by increasing SCC [7]. In the present study, LAP concentrations tended to decrease after colostrum administration in all groups in Experiment 2, although they did not differ significantly. This decrease may be attributable to the weakening of the inflammation status during the 7 days of the experiment. This is supported by the results that difference between before and after costrum administration was smaller than that in clinical mastitis. significantly higher than that in the subclinical group both before and after colostrum administration. $\mathrm{Na}$ is used as a parameter of inflammation in the mammary gland because it induces weak tight junctions between mammary epithelial cells. This allows the passage of $\mathrm{Na}$ in the blood between mammary epithelial cells, which increases the $\mathrm{Na}$ concentration in milk $[9,14]$. Therefore, clinical mastitis can cause more severe disruption of tight junctions, which increases $\mathrm{Na}$ concentration more than that in subclinical mastitis.

In experiment 2, $\mathrm{Na}$ concentration tended to decrease by colostrum administration in both $\mathrm{Cli}(-/+)$ groups, which is due to the recovery of tight junctions integrity between mammary epithelial cells as described above. However, experiment 1 
showed increased $\mathrm{Na}$ concentration by colostrum administration in spite of decreased

240 SCC. The reason of the difference between experiment 1 and 2 is not clear, although this 241 may be the tight junction repair delays compared with decreased SCC, due to the low 242 chemokine secretion. In Experiment 1, SCC was decreased in the milk after compared with before

244 colostrum administration, whereas a control group without colostrum was not prepared.

245 These results do not suggest that mastitis can be treated by oral administration of 246 colostrum. It was reported that when quarters with clinical mastitis caused by Escherichia coli intramammary infusion were assigned to groups with and without antibiotic administration for 5 days, there was a rapid reduction of SCC in both groups [2]. This report indicates that mastitis can be spontaneously cured without antibiotics. In experiment 2, SCC in both Cli (-/+) groups showed tendency to decrease, although there is no significant difference between before and after colostrum administration. These results may suggest that SCC is decreased spontaneously, regardless of colostrum. When mastitic milk was preserved at room temperature, the number of viable bacteria decreased

254 dramatically within a few hours, which was attributable to phagocytosis by neutrophils

$255[3,10]$. This report supports the possibility of spontaneous recovery from mastitis. In some cases for mastitis treatment, antibiotics was used together with antiinflammatory gel or other drugs. Therefore, further experiment is required to examine the effect of these treatments on the inflammation status of the udder under colostrum administration.

In conclusion, the present results suggest the possibility that oral colostrum

261 administration reduces inflammation in the mammary glands. Further studies are required 262 to confirm the effect of colostrum on treatment of clinical and subclinical mastitis without 

administration.

The authors declare that they have no conflicts of interest.

\section{References}

271

272 1. Bischoff-Kont, I. and Furst, R. 2021. Benefits of Ginger and Its Constituent 6-Shogaol 273 in Inhibiting Inflammatory Processes. Pharmaceuticals 14.

2742 2. Fuenzalida, M. J. and Ruegg, P. L. 2019. Short communication: Longitudinal study of 275 quarter-level somatic cell responses after naturally occurring, nonsevere clinical mastitis diagnosed 276 as culture negative, or caused by Escherichia coli or Klebsiella pneumoniae, and randomly assigned 277 to a no-treatment group or to receive intramammary ceftiofur. Journal of Dairy Science 102: $278 \quad 11476-11482$.

$2793 . \quad$ Hisaeda, K., Koshiishi, T., Watanabe, M., Miyake, H., Yoshimura, Y. and Isobe, N. 2016. 280 Change in viable bacterial count during preservation of milk derived from dairy cows with 281 subclinical mastitis and its relationship with antimicrobial components in milk. Journal of 282 Veterinary Medical Science 78: 1245-1250.

2834 4. Isobe, N. 2017. Control mechanisms for producing antimicrobial factors in ruminant 284 mammary gland. Animal Science Journal 88: 937-943.

285 5. Isobe, N., Hosoda, K. and Yoshimura, Y. 2009. Immunolocalization of lingual 286 antimicrobial peptide (LAP) in the bovine mammary gland. Animal Science Journal 80: 446-450.

287 6. Isobe, N., Nakamura, J., Nakano, H. and Yoshimura, Y. 2009. Existence of functional 288 lingual antimicrobial peptide in bovine milk. Journal of Dairy Science 92: 2691-2695.

289 7. Isobe, N., Morimoto, K., Nakamura, J., Yamasaki, A. and Yoshimura, Y. 2009. 290 Intramammary challenge of lipopolysaccharide stimulates secretion of lingual antimicrobial 291 peptide into milk of dairy cows. Journal of Dairy Science 92: 6046-6051.

292 8. Isobe, N., Matsukawa, S., Kubo, K., Ueno, K., Sugino, T., Nii, T. and Yoshimura, Y. 2020. 293 Effects of oral administration of colostrum whey in peripartum goat on antimicrobial peptides in 
postpartum milk. Animal Science Journal 91: 7. sodium, potassium, calcium, and electrical conductivity meters for diagnosing subclinical mastitis and intramammary infection in dairy cattle. Journal of Veterinary Internal Medicine 33: 23432982353.

299 10. Koshiishi, T., Watanabe, M., Miyake, H., Hisaeda, K. and Isobe, N. 2017. Cellular and 300 soluble components decrease the viable pathogen counts in milk from dairy cows with subclinical mastitis. Journal of Veterinary Medical Science 79: 1389-1393.

302 11. Matsukawa, S., Ueno, K., Sugino, T., Yoshimura, Y. and Isobe, N. 2018. Effects of 303 colostrum whey on immune function in the digestive tract of goats. Animal Science Journal 89: $304 \quad 1152-1160$.

305 12. Nishikawa, M., Nii, T., Yoshimura, Y. and Isobe, N. 2018. Investigation of the binding 306 of goat cathelicidin-7 to lipopolysaccharide and leucocidal suppression of pro-inflammatory 307 cytokines. Small Ruminant Research 168: 101-106.

308 13. Purba, F. Y., Nii, T., Yoshimura, Y. and Isobe, N. 2019. Short communication: 309 Production of antimicrobial peptide S100A8 in the goat mammary gland and effect of 310 intramammary infusion of lipopolysaccharide on S100A8 concentration in milk. Journal of Dairy 311 Science 102: 4674-4681.

312 14. Purba, F. Y., Ishimoto, Y., Nii, T., Yoshimura, Y. and Isobe, N. 2021. Effect of temporary 313 cessation of milking on the innate immune components in goat milk. Journal of Dairy Science 104: $314 \quad 10374-10381$.

315 15. Rees, A., Fischer-Tenhagen, C. and Heuwieser, W. 2017. Udder firmness as a possible 316 indicator for clinical mastitis. Journal of Dairy Science 100: 2170-2183.

317 16. Satyaraj, E., Reynolds, A., Pelker, R., Labuda, J., Zhang, P. F. and Sun, P. C. 2013.

318 Supplementation of diets with bovine colostrum influences immune function in dogs. British 319 Journal of Nutrition 110: 2216-2221.

320 17. Shibata, I., Ono, M. and Mori, M. 2001. Passive protection against porcine epidemic 321 diarrhea (PED) virus in piglets by colostrum from immunized cows. Journal of Veterinary Medical 322 Science 63: 655-658.

323 18. Shimazaki, K. and Kawai, K. 2017. Advances in lactoferrin research concerning bovine 324 mastitis. Biochemistry and Cell Biology 95: 69-75.

325 19. Zhang, G. W., Lai, S. J., Yoshimura, Y. and Isobe, N. 2014. Expression of cathelicidins 326 mRNA in the goat mammary gland and effect of the intramammary infusion of lipopolysaccharide 327 on milk cathelicidin-2 concentration. Veterinary Microbiology 170: 125-134.

328 20. Zhang, G. W., Lai, S. J., Yoshimura, Y. and Isobe, N. 2014. Messenger RNA expression 329 and immunolocalization of psoriasin in the goat mammary gland and its milk concentration after 
330 an intramammary infusion of lipopolysaccharide. Veterinary Journal 202: 89-93.

331 


\section{Figure legends}

Figure 1 Effect of oral administration of colostrum on somatic cell count (SCC), sodium (Na), immunoglobulin A (IgA), and lingual antimicrobial peptide (LAP) concentrations in milk of cows (Experiment 1). * denote significant difference between pre and post colostrum administration $(\mathrm{p}<0.05)$

Figure 2 Effect of oral administration of colostrum on udder firmness (A), somatic cell count (SCC) (B) and sodium (Na) concentration in bovine clinical and subclinical mastitis (Experiment 2). * denote significant difference between day 0 and day 7 of colostrum administration $(\mathrm{p}<0.05)$. ${ }^{\mathrm{a}, \mathrm{b}}$ denote significant

Figure 3 Effect of oral administration of colostrum on lingual antimicrobial peptide (LAP) (A) and immunoglobulin A (IgA) (B) concentrations in milk of cows with difference between different letters in each group on day 0 or day $7(p<0.05)$. clinical and subclinical mastitis (Experiment 2). * denote significant difference between day 0 and day 7 of colostrum administration $(p<0.05)$. $\quad{ }^{a, b}$ denote significant difference between different letters in each group on day 0 or day 7 
(a)

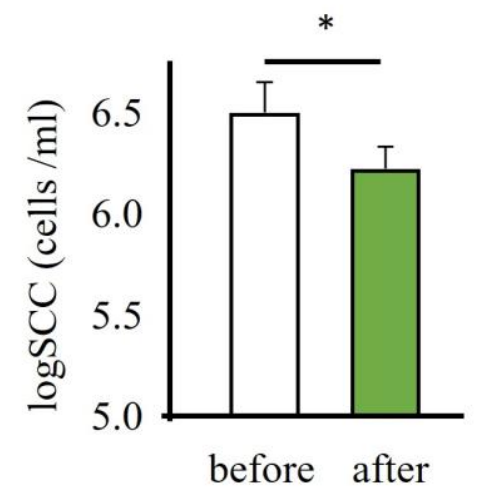

(c)

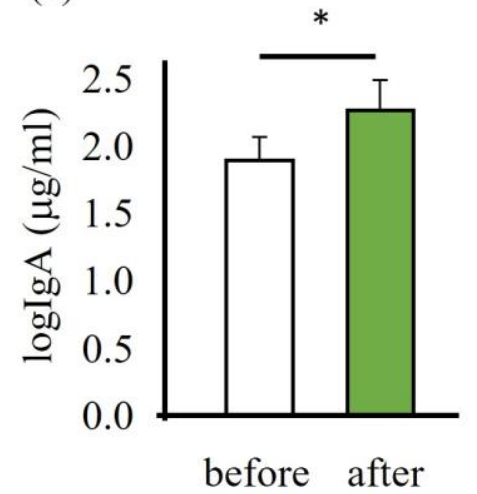

(b)

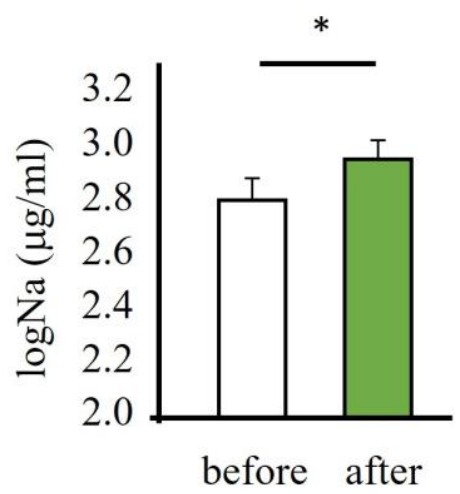

(d)

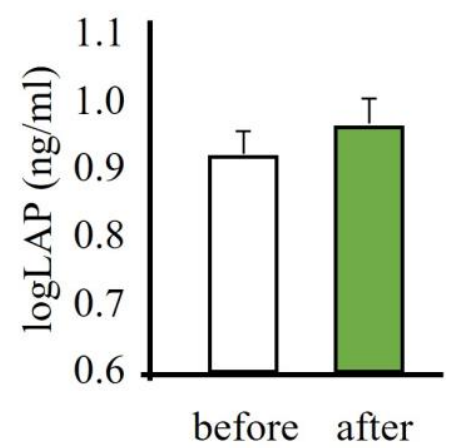

353

Fig. 1 


$$
\begin{array}{lll}
\text { (A) } & \text { Group: } & \mathrm{p}<0.001^{*} \\
& \text { Time: } & \mathrm{p}<0.001^{*} \\
& \text { Group } \times \text { Time: } & \mathrm{p}=0.317
\end{array}
$$

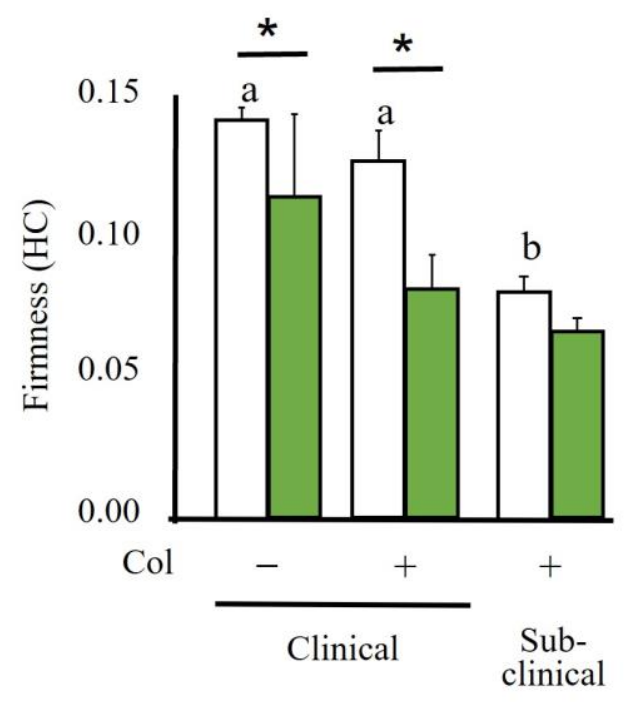

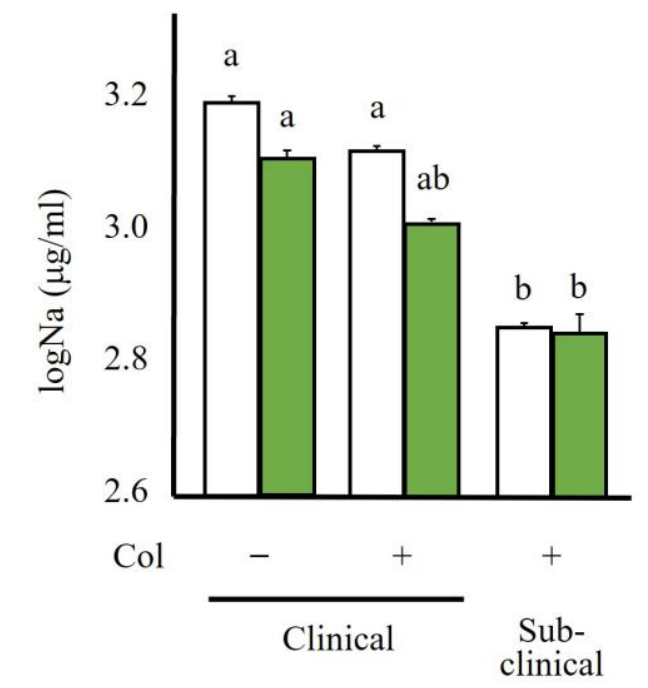

$360 \quad$ Fig. 2

361
(B) Group: $\quad \mathrm{p}=0.079$
Time: $\quad \mathrm{p}=0.096$
Group $\times$ Time: $\quad \mathrm{p}=0.813$

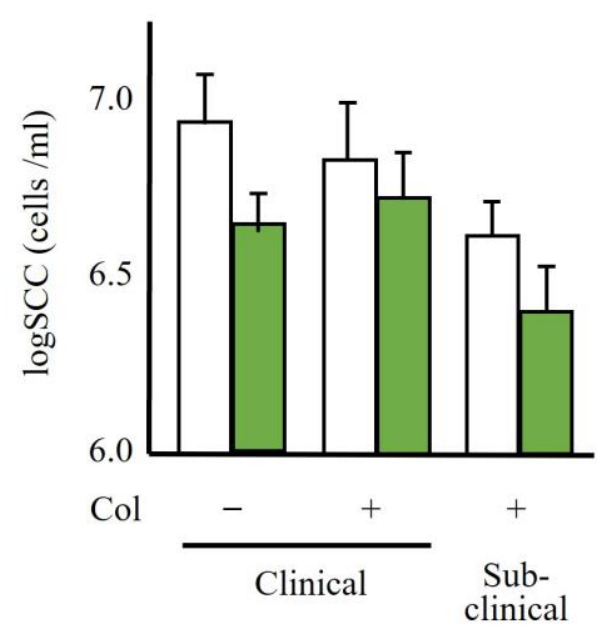

before administration

after administration 

(A) Group :
Time:

$$
\begin{aligned}
& p=0.057 \\
& p=0.002 * \\
& p=0.097
\end{aligned}
$$
Group $\times$ Time:

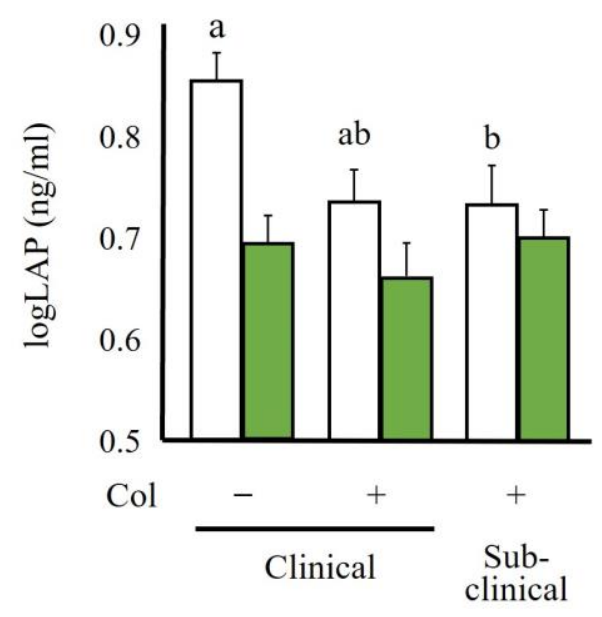

363

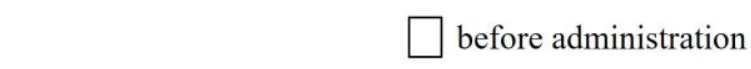
(B) Group: $\quad \mathrm{p}<0.001^{*}$ Time: $\quad \mathrm{p}=0.001$ * Group $\times$ Time: $\quad p=0.007 *$

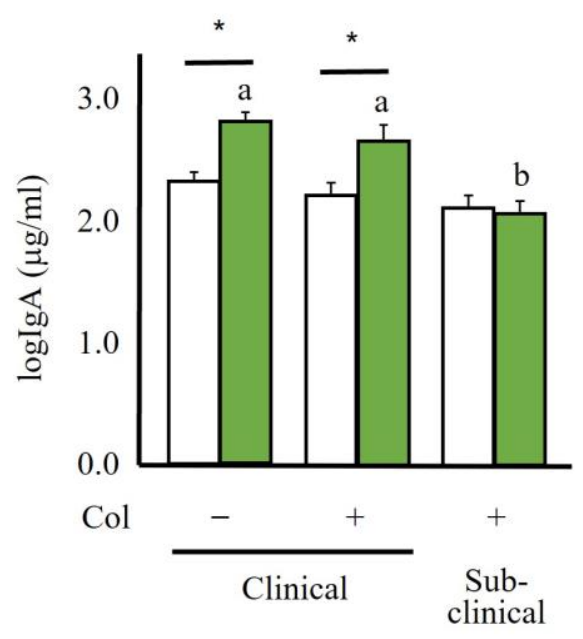

after administration

Fig. 3 